\title{
Contribuição da Doença e sua Terapêutica no Índice de Dano SLICC/ACR na Fase Precoce do Lúpus Eritematoso Sistêmico ${ }^{*}$
}

\author{
Contribution of the Disease and its Therapy on the SLICC/ACR-DI \\ in Early Systemic Lupus Erythematosus
}

\author{
Mailze Campos Bezerra ${ }^{(1)}$, Francisco Saraiva da Silva Júnior ${ }^{(1)}$, Eduardo Ferreira Borba Neto ${ }^{(2)}$ e Eloisa Bonfá ${ }^{(3)}$
}

\section{RESUMO}

A maioria das informações relativas ao curso da doença de pacientes com lúpus eritematoso sistêmico (LES) é restrita aos primeiros cinco anos após o diagnóstico. Entretanto, dados sobre a precocidade de instalação dos danos são pouco descritos. Objetivo: identificar os danos precoces de pacientes com LES de diagnóstico recente e verificar a influência da própria doença ou de sua terapêutica como principal causa para a instalação destes danos. Métodos: foram estudados retrospectivamente 82 pacientes com LES, cujo diagnóstico foi realizado de 1998 até 1999 em nosso serviço. Em todos os pacientes o escore do índice de dano do Systemic Lupus Internacional Collaborating Clinics/American College of Rheumatology-Damage Index (SLICC/ACR-DI) foi avaliado no momento do diagnóstico e anualmente três vezes consecutivas. As manifestações clínicas e o esquema terapêutico foram avaliados nas consultas médicas. O escore do SLICC/ACR para cada órgão/sistema e a prevalência dos danos foi identificado, assim como a sua possível causa (doença ou terapia). Resultados: A idade (média) inicial foi de 31,6 anos com predomínio do sexo feminino (94\%). A maioria dos pacientes (94\%) apresentou manifestações cutâneas e articulares, seguidas pelas renais (40\%), pulmonares (28\%) e neurológicas (18\%). Ao final do estudo, 52 pacientes $(63,4 \%)$ não apresentaram seqüelas (sem pontuação no SLICC). Dos 30 pacientes restantes, 16 tiveram escore $=1$ e 14 pacientes apresentaram escore $=2$. No total observamos 36 danos, sendo 23 atribuídos à doença e 13 à terapia (64\% vs 36\%, p < 0,05). Os sistemas mais freqüentemente acometidos foram o articular e o neurológico $(27,7 \%)$, seguidos pelo renal $(13,8 \%)$. Todos os pacientes com comprometimento neurológico tiveram danos precoces decorrentes da própria doença (apesar da terapêutica agressiva) enquanto a terapêutica com corticóide foi responsável pelos danos em $7 / 10$ pacientes com dano articular

\begin{abstract}
The majority of the information available about the disease course of patients with systemic lupus erythematosus (SLE) is restricted to the first five years after disease onset. However, data about early damage were rarely reported. Objective: to describe the early damage outcome of SLE patients in early disease and to discriminate the influence of disease itself or its therapy as the main cause of their damage. Methods: we retrospectively studied 82 patients with SLE, diagnosed during the period from 1998 to 1999 in our institution. In all patients the Systemic Lupus International Collaborating Clinics/American College of Rheumatology Damage Index (SLICC/ACR-DI) was scored at the diagnosis and annually for the next three years. Clinical manifestations were evaluated at each visit as well as the therapy. The SLICC/ACR-DI scores for each organ system and the prevalence of damage within organ systems were assessed, as well as its possible main cause (disease or therapy). Results: the mean age at entry was 31.6 years with female predominance of $94 \%$. The majority of the patients (94\%) presented cutaneous and articular involvement, $40 \%$ renal, 28\% pulmonary, and 18\% neurological manifestations. At the end of the study, 52 (63.4\%) patients had no damage to score with SLICC. From the remaining 30 patients, 16 had a score of 1 and 14 had a score $=2$. In total, 36 damages occurred, 23 of them attributed to the disease and 13 to the therapy (64\% vs. 36\%; $p<0.05)$. The most frequently injured systems were the articular and the neurological (27.7\%) ones, followed by the renal (13.8\%) system. All patients with neurological involvement have early damage due to the disease itself in spite of the aggressive therapeutic whereas from 10 patients with articular damage, $70 \%$ (7) were associated with steroid therapy (osteonecrosis). In contrast, premature renal injury
\end{abstract}

\footnotetext{
* Trabalho realizado na disciplina de reumatologia da Faculdade de Medicina da Universidade de São Paulo (FMUSP). Recebido em 5/6/2003. Aprovado, após revisão, em 8/1/2004.

1. Pós-graduando da disciplina de reumatologia da FMUSP.

2. Médico assistente da disciplina de reumatologia da FMUSP.

3. Professora titular da disciplina de reumatologia da FMUSP.

Endereço para correspondência: Eduardo Ferreira Borba Neto. Av. Dr. Arnaldo, 455, 3. andar, Reumatologia, sala 3.133, Cerqueira César, São Paulo, CEP 01246-903. E-mail:reumato@edu.usp.br
} 
(osteonecrose). O dano renal foi observado em 13,8\% dos pacientes (nefrite grave não responsiva ao tratamento). Outros efeitos adversos da terapia foram angina, catarata, diabetes e menopausa precoce. Conclusões: este estudo sugere que o dano precoce é ainda um importante problema no LES. São necessárias estratégias eficazes no sentido de minimizar as seqüelas, com destaque para as neurológicas. Redução da morbidade associada à terapia com corticóide também deve ser considerada utilizando-se agentes poupadores de esteróides.

Palavras-chave: lúpus eritematoso sistêmico, SLICC/ACR-DI, questionário, dano tecidual, tratamento.

\section{INTRODUÇÃO}

Grandes resultados no tratamento do lúpus eritematoso sistêmico (LES) foram obtidos nas últimas décadas. Podese verificar expressiva diminuição da taxa de mortalidade dos pacientes, sendo que atualmente a sobrevida em 20 anos é próxima de $70 \%{ }^{(1)}$. Apesar do gradativo avanço obtido em termos de redução da mortalidade, a morbidade decorrente da própria doença e da terapêutica passou a assumir maior importância no prognóstico dos pacientes.

Ante a necessidade de um instrumento padronizado para esta avaliação, em 1991 foi desenvolvido o Systemic Lupus Internacional Collaborating Clinics/American College of Rheumatology-Damage Index (SLICC/ACR-DI), índice de avaliação de danos de pacientes com LES ${ }^{(2)}$. Trata-se de um instrumento desenvolvido especificamente para quantificar prejuízos irreversíveis no $\mathrm{LES}^{(2-4)}$, que se mostrou reprodutível e validado para esta finalidade ${ }^{(2-3)}$. Neste sentido, este índice é utilizado atualmente em estudos longitudinais e trials terapêuticos ${ }^{(4-7)}$, pois mensura as seqüelas resultantes tanto da doença quanto de seu tratamento, de forma cumulativa desde o seu início(2).

Apesar de seu valor prognóstico e de incluir uma ampla série de prejuízos permanentes dos diversos órgãos e sistemas, este índice não discrimina aqueles decorrentes especificamente da doença e/ou de sua terapia ${ }^{(2)}$. Neste sentido, este estudo se propõe a avaliar as repercussões da terapia e da própria doença em pacientes com diagnóstico precoce através do SLICC/ACR.

\section{PACIENTES E MÉTODOS}

Foram analisados retrospectivamente os prontuários de 82 pacientes com diagnóstico de LES pelos critérios do ACR entre os anos de 1998 e $1999^{(8)}$, atendidos no ambulatório da disciplina de reumatologia do Hospital das Clínicas da was observed in $13.8 \%$, all of them with unresponsive severe nephritis. Other adverse effect of therapy included 1 angina, 2 cataracts, 1 diabetes and 2 premature menopauses. Conclusions: this study suggests that early damage is still a major problem in SLE that reinforces the need for new strategies in order to minimize injury, particularly for neurological manifestations. Moreover, it also seems important to reduce morbidity induced by corticosteroid therapy with the use of steroid-sparing agents.

Keywords: systemic lupus erythematosus, damage, SLICC/ACR-DI, therapy.

Faculdade de Medicina da Universidade de São Paulo (HC/FMUSP), no período de junho a dezembro de 2001. O protocolo foi aprovado pelo comitê de ética dessa instituição.

Os parâmetros clínicos analisados para o envolvimento sistêmico foram os estabelecidos para os critérios de classificação do $\operatorname{LES}^{(8)}$ incluindo artralgia para o envolvimento articular, lesão máculo-papular eritematosa em áreas expostas ou outra lesão definida como associada ao lúpus e confirmada por biópsia para o quadro cutâneo, pneumonite intersticial confirmada por tomografia computadorizada para o envolvimento pulmonar, miocardite confirmada por eletrocardiograma (ECG) e ecocardiograma para o envolvimento cardíaco. Acidente vascular cerebral, neuropatia periférica e mielite transversa, definidos como associados ao lúpus para o quadro neurológico.

A avaliação do índice de dano SLICC/ACR foi realizada de acordo com os critérios estabelecidos ${ }^{(2)}$ e validados ${ }^{(3)}$. Os dados foram obtidos retrospectivamente durante a consulta e através de extensa revisão dos prontuários. Estes parâmetros foram definidos para o momento do diagnóstico e anualmente até completar três anos de seguimento.

Os exames complementares avaliados neste trabalho foram os estabelecidos nos critérios de classificação do $\operatorname{LES}^{(8)}$ acrescidos daqueles mencionados acima e dos definidos para avaliação do índice de dano ${ }^{(2)}$, ressaltando-se que a disfunção cognitiva foi documentada apenas por exame clínico. Todos os soros foram também testados para a presença de anticorpos anti-cardiolipina da classe $\operatorname{IgG}$ e $\operatorname{IgM}$, pelo método de ELISA ${ }^{(9)}$.

Análise estatística foi realizada através do teste não paramétrico qui-quadrado para a comparação de proporções entre os grupos estudados. O nível de significância adotado foi de $5 \%$. 


\section{RESULTADOS}

Entre os 82 pacientes analisados houve predomínio do sexo feminino (77 pacientes, 94\%), sendo a média de idade no diagnóstico de 31,6 anos (variando de 21,3 a 41,9 anos).

As freqüências de manifestações clínicas estão ilustradas na Tabela 1. A maioria dos pacientes apresentou quadro articular e cutâneo (94\%) e 62\% apresentaram envolvimento hematológico. O acometimento renal ocorreu em 33 pacientes (40\%), enquanto o quadro neurológico só foi observado em 15 pacientes.

Do total de pacientes analisados, 30 (37\%) apresentaram algum dano no período de até três anos de observação quando avaliados pelo índice de dano SLICC/ACR, num total de 36 eventos, analisados de acordo com sua possível relação causal, isto é, associados à doença ou conseqüentes à terapia (Tabela 2). Os sistemas mais afetados por estes danos foram o neurológico e o articular (28\%), seguido pelo renal (14\%).

Das 15 pacientes com envolvimento neurológico, 10 apresentaram lesões permanentes, unicamente associadas à atividade da doença (Tabela 2). Houve predomínio das seqüelas relacionadas com quadros focais, representados por cinco casos de neuropatia periférica, quatro casos de acidente vascular cerebral e um caso de mielite transversa. Os cinco casos com quadro neurológico difuso não evoluíram com danos permanentes (Tabela 1). Dois pacientes com quadro de convulsão não pontuaram pelo índice, pois não necessitaram terapia anticonvulsivante por mais de seis meses. Três pacientes apresentaram psicose de duração limitada, necessitando terapia por período muito inferior a seis meses. Não observamos disfunção cognitiva.

O envolvimento renal, complicação em 33 pacientes, resultou em lesão permanente significativa em $14 \%$ dos casos: três pacientes com proteinúria $>3,5 \mathrm{~g} / \mathrm{dia}$, um paciente com taxa de filtração glomerular $<50 \%$ e um com insuficiência renal terminal (Tabelas 1 e 2). Os cinco casos de dano renal foram decorrentes de seqüelas exclusivamente relacionadas com atividade de nefrite grave (Tabela 2).

Com relação ao sistema músculoesquelético houve dano em 10/77 casos (12,98\%) (Tabela 1), sendo relacionado com terapia em $70 \%$ dos pacientes (Tabela 2). Oito pacientes apresentaram osteonecrose, sendo $3 \mathrm{em}$ múltiplos sítios (não foram relatados como mais de um evento). Dos 8 pacientes com osteonecrose, 3 ocorreram no primeiro ano, 3 no segundo e 2 no terceiro ano. Interessante notar que todos estavam sob corticoterapia com doses superiores
TABELA 1

MANifESTAÇÕES CLÍNICAS EM 82 PACIENTES COM DIAGNÓSTICO RECENTE DE LES

\begin{tabular}{lc}
\hline Manifestação & \# Positivo (\%) \\
\hline Articulares & $77(94 \%)$ \\
\hline Dermatológicas & $77(94 \%)$ \\
Hematológicas & $51(62 \%)$ \\
Renais & $33(40 \%)$ \\
Pulmonares & $23(28 \%)$ \\
Pleurite & 22 \\
Pneumonite & 1 \\
Cardiológicas & $13(16 \%)$ \\
Pericardite & 11 \\
Miocardite & 2 \\
Neurológicas & $15(18 \%)$ \\
Convulsão & 2 \\
Psicose & 3 \\
AvC & 4 \\
Neuropatia Periférica & 5 \\
Mielite Transversa & 1 \\
\hline
\end{tabular}

TABELA 2

ANÁLISE dOS 36 EVENTOS DE DANOS E SUA POSSÍVEL ASSOCIAÇÃO CAUSAL EM 30 PACIENTES COM LES RECENTE

\begin{tabular}{lccc}
\hline Órgão/Sistema & $\begin{array}{c}\text { Total } \\
\text { danos } \\
\mathbf{N}(\%)\end{array}$ & $\begin{array}{c}\text { Dano por } \\
\text { doença } \\
\mathbf{N}(\%)\end{array}$ & $\begin{array}{c}\text { Dano por } \\
\text { terapia } \\
\mathbf{N}(\%)\end{array}$ \\
\hline Neurológico & $10(28 \%)$ & $10(43 \%)$ & 0 \\
\hline Articular & $10(28 \%)$ & $3(13 \%)$ & $7(54 \%)$ \\
\hline Renal & $5(14 \%)$ & $5(22 \%)$ & 0 \\
\hline Vascular periférico & $4(10 \%)$ & $4(17 \%)$ & 0 \\
\hline Ocular & $2(5 \%)$ & 0 & $2(15 \%)$ \\
\hline Falência ovar. prematura & $2(5 \%)$ & 0 & $2(15 \%)$ \\
\hline Pulmonar & $1(3 \%)$ & $1(4 \%)$ & 0 \\
\hline Cardíaco & $1(3 \%)$ & 0 & $1(8 \%)$ \\
Diabetes & $1(3 \%)$ & 0 & $1(8 \%)$ \\
\hline Dermatológico & 0 & 0 & 0 \\
Malignidade & 0 & 0 & 0 \\
Gastrointestinal & 0 & 0 & 0 \\
\hline Total & 36 & 23 & 13 \\
\hline
\end{tabular}

a $30 \mathrm{mg} /$ dia no primeiro ano de seguimento e 5 permaneceram com esta dose também no segundo ano. Apenas um paciente apresentou anticorpo anticardiolipina positivo em títulos baixos no início e posteriormente em altos títulos, associado à perda de polpa digital, sendo considerado como 
síndrome do anticorpo anti-fosfolipídeo (SAAF), sendo que neste caso a osteonecrose foi provavelmente atribuída à própria doença. Todos os casos de osteonecrose eram sintomáticos e o diagnóstico inicial foi realizado por radiologia simples. Apesar do curto tempo de doença, de apenas três anos, foi observada artrite deformante redutível em dois pacientes.

Outros efeitos adversos atribuídos à terapia com corticosteróide foram catarata (dois casos) e diabetes (um caso). Além disso, uma paciente apresentou infarto agudo do miocárdio, necessitando revascularização miocárdica, porém não possuía outros fatores de risco para doença coronariana como hipertensão arterial, dislipidemia, antifosfolípides (Tabela 2). Duas pacientes apresentaram falência ovariana prematura (FOP), com idade de 35 e 39 anos, provavelmente relacionada com maior tempo de uso de ciclofosfamida.

Com relação a outros danos associados à doença observamos um caso de fibrose pulmonar e quatro casos associados ao sistema vascular periférico: perda de polpa digital (dois casos), amputação de metatarsos (um caso) e trombose venosa profunda (um caso).

Nenhum paciente apresentou lesões permanentes decorrentes do quadro dermatológico ou gastrointestinal, bem como não foram diagnosticados casos de malignidade no período do estudo (Tabela 2).

Avaliação global dos 36 eventos de danos permanentes mostrou que o dano induzido pela terapêutica é significativamente menor que aquele causado pela doença (Tabela 2). Dos danos, 64\% foram atribuídos à atividade da doença, enquanto $36 \%$ foram decorrentes da terapia $(\mathrm{p}=0,04)$. É interessante notar que a maioria das seqüelas (56\%) relacionadas com a atividade da doença foi identificada no momento do diagnóstico (quatro danos) e no fim do primeiro ano da doença (nove danos). Da mesma forma, a maioria dos danos por terapêutica (77\%) ocorreu nos primeiros dois anos com cinco danos ao final do primeiro ano e cinco no final do segundo ano.

A análise da evolução do índice de dano no período de três anos nos 82 pacientes demonstrou que, no momento do diagnóstico, apenas 7\% dos pacientes já apresentavam lesão permanente, com índice de dano médio/paciente de 0,073 (Tabela 3). Houve uma significativa progressão gradativa de aparecimento de lesões permanentes no grupo e de acúmulo de danos em pacientes individualmente, resultando no final de três anos em mais de um terço dos pacientes com lesão permanente (37\%). Destes, $17 \%$ acumularam mais de um dano. Houve também um aumento de cerca de oito vezes no índice de dano médio/paciente $(0,6)$ ao final do período estudado.
TABELA 3

PROGRESSÃO EM TRÊS ANOS DA FREQÜÊNCIA DO ÍNDICE DE DANO (SLICC) EM 82 PACIENTES COM LES RECENTE

\begin{tabular}{lcccc}
\hline & $\begin{array}{c}\text { Ano 0 } \\
\text { (no diagnóstico) } \\
\text { n (\%) }\end{array}$ & Ano 1 & Ano 2 & Ano 3 \\
\hline SLICC 0 & $76(93 \%)$ & $62(76 \%)$ & $57(69 \%)$ & $52(63 \%)$ \\
SLICC 1 & $6(7 \%)$ & $15(18 \%)$ & $14(17 \%)$ & $16(19 \%)$ \\
SLICC 2 & 0 & $4(5 \%)$ & $10(12 \%)$ & $9(11 \%)$ \\
SLICC 3 & 0 & $1(1 \%)$ & $1(1 \%)$ & $5(6 \%)$ \\
SLICC MÉDI0 & 0,073 & 0,317 & 0,451 & 0,597 \\
\hline
\end{tabular}

\section{DISCUSSÃO}

Este estudo mostra que, apesar da maioria dos casos de dano precoce estar diretamente associada à atividade da doença (com destaque para o sistema nervoso), mais de um terço dos eventos de lesão permanente são decorrentes da terapêutica. Observamos ainda que a evolução dos danos é rapidamente progressiva ao longo de três anos após o diagnóstico.

O envolvimento focal do sistema nervoso e o quadro renal foram as principais causas de danos associados à doença. Já foi demonstrado que manifestações neurológicas focais (acidente vascular cerebral, convulsão, neuropatia, mielite transversa) estão relacionadas com um pior prognóstico, com elevada mortalidade em 5, 10 e 20 anos quando comparados com pacientes com sintomas neuropsiquiátricos difusos (cefaléia, síndrome orgânica cerebral, psicose $)^{(10)}$. Dados da literatura mostram que o dano no início da doença está relacionado com maior mortalidade ${ }^{(12)}$.

Ressalta-se que o dano neurológico precoce continua sendo um problema significativo no LES, visto que mais da metade dos pacientes com acometimento neurológico $(66 \%)$ evoluíram com lesão permanente ${ }^{(13,14)}$. Em contraste, apenas 15\% dos casos de envolvimento renal apresentaram lesão permanente, o que estaria de acordo com a freqüência de casos refratários ao tratamento padrão com ciclofosfamida ${ }^{(15)}$, reforçando que, apesar de uma resposta muito superior que a do quadro neurológico, ainda existe a necessidade de se buscar alternativas terapêuticas para um subgrupo mais grave de lesão renal ${ }^{(16)}$.

Outros danos associados à doença foram: o vascular periférico (três casos de vasculite provocando perdas menores de substâncias e uma amputação); e um caso de trombose venosa profunda associado à SAAF. A artropatia 
de Jaccoud ocorre em 3\% a 38\% dos casos de LES $^{(17-21)}$ e é considerada uma deformidade tardia do envolvimento articular. Dois casos apresentaram o padrão típico com subluxações, sem erosões, no primeiro ano de doença.

As lesões permanentes decorrentes do uso de drogas ocorreram em um número significativo de pacientes (39\%), e para a maioria dos casos esteve associada ao uso de corticosteróide.

Neste aspecto, a principal causa de dano para o sistema músculoesquelético esteve relacionada com necrose asséptica. Este quadro foi inicialmente descrito em 1960 por Dubois e Cozen ${ }^{(22)}$, com prevalência estimada de apenas $5 \%$. Estudos mais recentes têm documentado prevalências variando de $14 \%$ a $40 \%$, provavelmente resultantes de melhores métodos diagnósticos ${ }^{(23)}$.

A terapêutica com corticosteróide tem sido relatada como a principal causa de osteonecrose $\mathrm{e}^{(24,25)}$. De fato, foi demonstrado que o uso destas drogas leva a uma redução de formação e turnover ósseo. Adicionalmente foi observado um aumento da apoptose do osteócito ${ }^{(24)}$. Outros fatores também têm sido implicados na patogênese desta complicação, como a SAAF ${ }^{(26,27)}$. Além disso, foi observada a associação da presença de anticorpos anticardiolipina e de osteonecrose de fềmur não traumática em pacientes sem diagnóstico de $\operatorname{LES}^{(28)}$. Neste estudo, apenas uma paciente com esta complicação apresentava anticorpos anticardiolipina. A mesma apresentou perda de polpa digital atribuída à SAAF, sendo este dano atribuído à doença. Desta forma, a maioria dos casos de osteonecrose identificados em nossa população parece estar relacionada diretamente com a corticoterapia, visto que cinco dos oito pacientes foram mantidos com altas doses por pelo menos dois anos de seguimento e nenhum deles possuía anticorpos anticardiolipina.

\section{REFERÊNCIAS}

1. Abu-Shakra, Urowitz MB, Gladman DD, Gough J: Mortality studies in systemic lupus erytematosus. Results from a single centre. II. Predictor variables for mortality. J Rheumatol 22: 1265-70, 1995.

2. Gladman DD, Ginzler E, Goldsmith C, et al: The development and initial validation of the systemic lupus international collaborating clinics/American College of Rheumatology damage index for systemic lupus erythematosus. Arthritis Rheum 39: 363-9, 1996.

3. Gladmann DD, Urowitz MB, Goldsmith $\mathrm{CH}$, et al: The reliability of the systemic lupus international collaborating clinics/American College of Rheumatology damage index in patients with systemic lupus erythematosus. Arthritis Rheum 40: 809-13, 1997.

4. Gladman DD, Urowitz MB. The SLICC/ACR damage index: progress report and experience in the field. Lupus 8: 632-7, 1999.
Outros danos associados ao uso de corticoteróides foram diabetes, catarata e infarto agudo do miocárdio. Com relação a esta última complicação, observa-se que a incidência de doença coronariana em LES está aumentando nos últimos anos e estudo epidemiológico recente demonstrou que o risco em mulheres jovens com LES é 50 vezes maior que na população normal ${ }^{(29)}$.

A falência ovariana prematura tem sido diretamente associada ao uso de alquilantes, particularmente a ciclofosfamida, estando relacionada com a dose cumulativa, duração da terapia e a idade da paciente ${ }^{(30)}$. Neste aspecto, as duas pacientes com esta manifestação iniciaram a terapia com ciclofosfamida com 35 e 39 anos de idade, sendo a FOP provavelmente relacionada com o maior tempo de uso da ciclofosfamida.

A eficácia do índice de SLICC de detectar mudanças ao longo do tempo já foi demonstrada em estudo prévio ${ }^{(31)}$ e confirmada neste estudo. O aumento do dano paralelo à duração da doença nos primeiros anos amplia observações anteriores que após dez anos da doença, 70\% dos pacientes apresentavam dano avaliado pelo SLICC/ACR ${ }^{(5)}$.

Estudos com acompanhamento deste índice nos pacientes em 2 anos, 5-10 anos e mais de 10 anos mostraram que a progressão de 2 anos até 5-10 anos de doença é mais lenta que o observado neste trabalho com uma média de progressão de 2,3 vezes $^{(12)}$.

Concluímos que o dano é rapidamente progressivo nos primeiros anos da doença. Enfatizamos que são necessárias novas estratégias terapêuticas no sentido de minimizar as seqüelas decorrentes da doença, em particular do sistema neurológico. Outro aspecto importante é a necessidade de se reduzir a morbidade induzida pela terapêutica, que tem como principal enfoque a avaliação do risco/benefício de se utilizar, quando possível, agentes poupadores de corticosteróide.

5. Zonna-Nacach A, Camargo-Coronel A, Yanez P, et al: Measurement of damage in 210 mexican patients with systemic lupus erythematosus: relationship with disease duration. Lupus 7 : 119-23, 1998.

6. Nossent JC: SLICC/ACR damage index in afro-Caribbean patients with systemic lupus erythematosus: changes in and relationship to disease activity, corticosteroid therapy and prognosis. J Rheumatol 25: 654-9, 1998.

7. Stoll T, Stucki G, Malik J, et al: Association of the systemic lupus international collaborating clinics/American College of Rheumatology damage index with measures of disease activity and health status in patients with systemic lupus erythematosus. J Rheumatol 24: 309-13, 1997.

8. Tan EM, Chen AS, Fries JF, et al: The 1982 revised criteria for the classification of systemic lupus erythematosus. Arthritis Rheum 25: 1271-7, 1982. 
9. Gharavi AE, Harris EM, Asherson RA, Hughes GRV: Anticardiolipin antibodies isotype distribution and phospholipid specificity. Ann Rheum Dis 46: 1-6, 1987.

10. Kovacs JAJ, Urowitz MB, Gladman DD: Dilemmas in neuropsychiatric lupus. Rheum Dis Clin North Am 19: 795-813, 1993.

11. Stoll T, Seifert B, Isenberg DA: SLICC/ACR damage index is valid and renal e pulmonary organ scores are predictors of severe outcome in patients with systemic lupus erythematosus. Br J Rheumatol 35: 248-54, 1996

12. Gladman DD, Goldsmith CH, Urowitz MB, et al: The Systemic Lupus International Collaborating Clinics / American College of Rheumatology ( SLICC/ ACR) Damage Index for Systemic Lupus Erytematosus International Comparison. J Rheumatol 27: 373-6, 2000.

13. Borba EF, Bonfá E: A first step toward solving the neuropsychiatric lupus puzzle. J Clin Rheumatol 5: 312-3, 1999.

14. Sanni G, Khamashta MA: Low-dose pulse cyclophosphamide in the treatment of neuropsychiatric lupus. Lupus 12: 1-2, 2003.

15. Boumpas DT, Austin HA, Vaughn EM, et al: Controlled trial of pulse methylprednisolone versus two regimens of pulse cyclophosphamide in severe lupus nephritis. Lancet 340: 741-5, 1992.

16. Gescuk BD, Davis JC: Novel therapeutic agents for systemic lupus erythematosus. Curr Opin Rheumatol 14:515-21, 2002.

17. Alarcon-Segovia D, Abud-Mendonza C, Diaz-Jouanen E, et al: Deforming arthropathy of the hands in systemic lupus erythematosus. J Rheumatol 15: 65-9, 1988.

18. Bleifeld CJ, Inglis AE: The hand in systemic lupus erythematosus. J Bone Joint Surg 56A: 1207-15, 1974.

19. Dreyfus JN, Schnitzer TJ: Pathogenesis and differential diagnosis of the swan-neck deformity. Semin Arthritis Rheum 13: 200-11, 1983.

20. Esdaile JM, Danoff D, Rosenthal L, et al: Deforming arthritis in systemic lupus erythematosus. Ann Rheum Dis 40: 124-6, 1981.
21. Kahn MF: Jaccoud's syndrome in a rheumatology unit. Clin Rheum Pract Winter: 153-5, 1986.

22. Dubois EL, Cozen L: Avascular (aseptic) bone necrosis associated with lupus erythematosus. J Am Med Assoc 174: 966-84, 1960.

23. Petri M: Musculoskeletal complications of systemic lupus erythematosus in the Hopkins Lupus Cohort: an update. Arthritis Care Res 8: 137-45, 1995.

24. Manolagas SC, Weinstein RS: New developments in the pathogenesis and treatment of steroid-induced osteoporosis. J Bone Miner Res 14: 1061-6, 1999.

25. Cunnane G, Lane NE: Steroid-induced osteoporosis in systemic lupus erythematosus. Rheum Dis Clin North Am 26: 311-27, 2000.

26. Mont MA, Glueck CJ, Pacheco IH, Wang P, Hungerford DS, Petri M: Risk factors for osteonecrosis in systemic lupus erythematosus. J Rheumatol 24: 654-62, 1997.

27. Egan RM, Munn RK: Catastrophic antiphospholipid antibody syndrome presenting with multiple thromboses and sites of avascular necrosis. J Rheumatol 21: 2376-9, 1994.

28. Korompilias AV, Gilkeson GC, Ortel TL, Seaber AV, Urbaniak JR: Anticardiolipin antibodies and osteonecrosis of the femoral head. Clin Orthop 345: 174-80, 1997.

29. Manzi S, Meilahn EM, Rairie JE, et al: Age-specific incidence rates of myocardial infarction and angina in women with systemic lupus erythematosus. Am J Epidemiol 145: 408-15, 1997.

30. Boumpas DT, Austin HA 3rd, Vaughan EM, Yarboro CH, Klippel $\mathrm{J}$, Balow JE: Risk for sustained amenorrhea in patients with systemic lupus erythematosus receiving intermittent pulse cyclophosphamide therapy. Ann Intern Med 119: 366-9, 1993.

31. Bootsma H, Derksen RHWM, Jaegers SMHJ, et al: Usefulness of the SLICC/ACR damage index in patients with systemic lupus erythematosus. Arthritis Rheum 40(supp19): S160, 1997. 